\title{
Multiple cancer-specific antigens are targeted by a chimeric antigen receptor on a single cancer cell
}

\author{
Yanran He, ${ }^{1}$ Karin Schreiber, ${ }^{2}$ Steven P. Wolf, ${ }^{2}$ Frank Wen, ${ }^{2}$ Catharina Steentoft, ${ }^{3}$ Jonathan Zerweck, ${ }^{2}$ \\ Madeline Steiner, ${ }^{2}$ Preeti Sharma, ${ }^{4}$ H. Michael Shepard, ${ }^{5}$ Avery Posey, ${ }^{6,7}$ Carl H. June, ${ }^{7}$ \\ Ulla Mandel, ${ }^{3}$ Henrik Clausen, ${ }^{3}$ Matthias Leisegang, ${ }^{8}$ Stephen C. Meredith, ${ }^{2}$ David M. Kranz, ${ }^{4}$ \\ and Hans Schreiber ${ }^{1,2,9}$ \\ 'Committee on Cancer Biology, and 'Department of Pathology, The University of Chicago, Chicago, Illinois, USA. \\ ${ }^{3}$ Copenhagen Center for Glycomics, University of Copenhagen, Copenhagen, Denmark. ${ }^{4}$ Department of Biochemistry, \\ University of Illinois, Urbana, Illinois, USA. ${ }^{5}$ Biooncology Consultants, San Diego, California, USA. ${ }^{6}$ Department of Systems \\ Pharmacology and Translational Therapeutics, University of Pennsylvania, Philadelphia, Pennsylvania, USA. ${ }^{7}$ Center for \\ Cellular Therapies, Perelman School of Medicine, University of Pennsylvania, Philadelphia, Pennsylvania, USA. ${ }^{8}$ Institute \\ of Immunology, Charité - Universitätsmedizin Berlin, Campus Buch, Berlin, Germany. ${ }^{9}$ Committee on Immunology, The \\ University of Chicago, Chicago, Illinois, USA
}

Conflict of interest: $\mathrm{HS}$ and $\mathrm{YH}$ are inventors of intellectual property (IP) surrounding 237Ab-derived CARs. DMK has ownership interest in Bellicum Pharmaceuticals, Agenus Inc., and Jounce Therapeutics and is a consultant/advisory board member for AbbVie. DMK and PS are coinventors of the IP surrounding 237Ab-derived CARs. CH] reports research funding from Novartis, and he is a scientific founder of Tmunity Therapeutics, for which he has founder's stock but no income. $\mathrm{CH}$ also works under a research collaboration involving the University of Pennsylvania and the Novartis Institutes of Biomedical Research, Inc, and he is an inventor of IP licensed by the University of Pennsylvania to Novartis. ADP reports research funding from Tmunity Therapeutics around the clinical development of 5E5-CART cells and has IP licensed to Novartis for CART cell therapy as well as gene therapy. $\mathrm{HC}, \mathrm{CS}$, and UM are coinventors of IP surrounding 5E5Ab-derived CARs licensed by the University of Copenhagen to Novartis.

Copyright: () 2019, American Society for Clinical Investigation.

Submitted: May 22, 2019

Accepted: September 11, 2019

Published: November 1, 2019

Reference information: /CI Insight. 2019;4(20):e130416.

https://doi.org/10.1172/jci.

insight.130416.
Human cancer cells were eradicated by adoptive transfer of T cells transduced with a chimeric antigen receptor (CAR) made from an antibody (237Ab) that is highly specific for the murine Tnglycosylated podoplanin (Tn-PDPN). The objectives were to determine the specificity of these CARtransduced T (CART) cells and the mechanism for the absence of relapse. We show that although the 237Ab bound only to cell lines expressing murine Tn-PDPN, the 237Ab-derived 237CART cells lysed multiple different human and murine cancers not predicted by the 237Ab binding. Nevertheless, the 237CART cell reactivities remained cancer specific because all recognitions were dependent on the Tn glycosylation that resulted from COSMC mutations that were not present in normal tissues. While Tn was required for the recognition by 237CART, Tn alone was not sufficient for 237CART cell activation. Activation of 237CART cells required peptide backbone recognition but tolerated substitutions of up to 5 of the 7 amino acid residues in the motif recognized by 237Ab. Together, these findings demonstrate what we believe is a new principle whereby simultaneous recognition of multiple independent Tn-glycopeptide antigens on a cancer cell makes tumor escape due to antigen loss unlikely.

\section{Introduction}

Substantial experimental and clinical evidence indicates that the specificity of an antibody $(\mathrm{Ab})$ predicts the reactivity of the chimeric antigen receptor (CAR) that is derived from it. For example, anti-CD19 CAR T (CART) cells faithfully recapitulated the reactivity of the anti-CD19 Ab. While adoptive transfer of antiCD19 CART cells effectively treated patients with advanced CD19-positive B cell malignancies (1), the loss of CD19 from the cancer cell surface is a common cause of relapse (1). Several approaches now aim at targeting multiple different antigens simultaneously to avoid escape by antigen-loss variants (ALVs) (2-4). However, this strategy has been severely limited by the few cell surface antigens that can be targeted safely. Here, we show that even though an $\mathrm{Ab}(237 \mathrm{Ab}$; ref. 5) that is highly specific for the murine Tn-glycosylated podoplanin (Tn-PDPN) bound only to cells expressing Tn-PDPN (6), the 237CART cells derived from $237 \mathrm{Ab}$ cross-reacted with multiple different human cancers, as long as the cells carry a cancer-specific mutation that causes loss of COSMC function. This makes the extended reactivity still exclusively cancer specific, which makes 237CART cells an attractive candidate for treating Tn-glycosylated human cancers.

\section{Results}

To compare the efficacy in vivo of 237CART cells with that of anti-CD19CART treatment $(7,8)$, we used the human leukemia Jurkat cell line, which naturally carries a mutation that causes loss of COSMC func- 


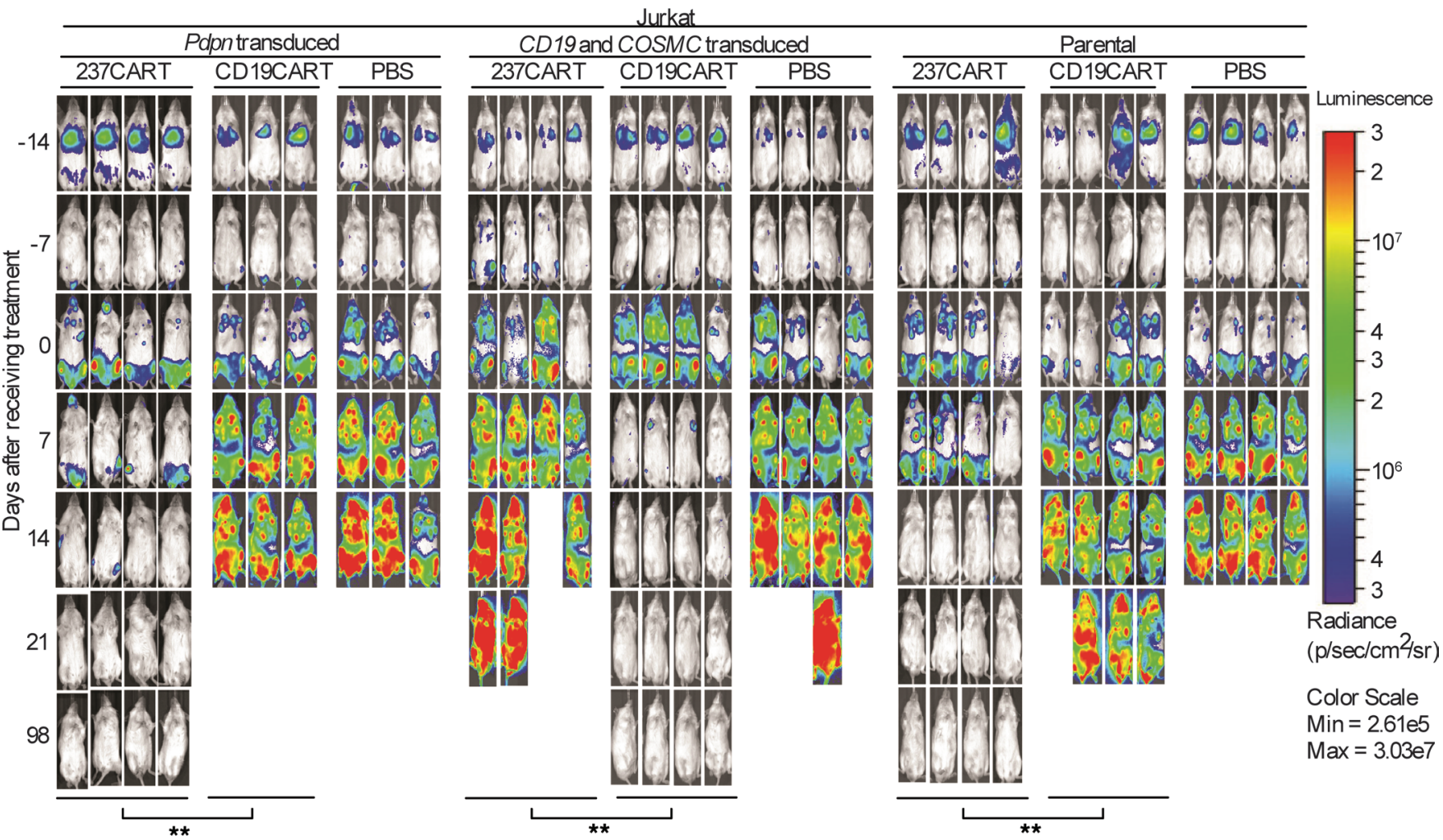

Figure 1. 237CART cells eradicate Tn-positive human cancers regardless of murine Tn-PDPN expression. Survival of NSG mice bearing 2-week-old established systemic Jurkat leukemia (Pdpn-, COSMC-, and CD19-transduced, and the parental cell line) that were treated with $5 \times 10^{6} 237$ CART cells, $5 \times 10^{6}$ CD19CART cells, or an equal volume of PBS ( $n=3$ or 4 mice per treatment group from at least 3 independent experiments as indicated in the figure). Disease progression was monitored weekly by bioluminescent imaging. The survival difference of the animals between the 2 groups was analyzed by log-rank Mantel-Cox test. ${ }^{* *} P \leq 0.01$.

tion. Jurkat was transduced to express murine PDPN, because previous work has shown that 237Ab strictly recognizes a 7-amino-acid-residue-long epitope surrounding the Tn-glycosylated $\mathrm{Thr}^{77}$ of murine PDPN (6), while only 1 of the 7 amino acid residues within the 237Ab epitope is conserved between murine and human PDPN (9). Figure 1 shows that 237CART cells eradicated a systemic burden of murine PDPN-transduced Jurkat leukemia cells and achieved a long-term survival free of relapse. The efficacy was comparable to that of CD19CART cell treatment of Jurkat cells by targeting surface CD19 expression (Figure 1). Given that 237Ab does not recognize Jurkat cells without murine PDPN expression (Figure 2), it was surprising that 237CART cells also eradicated the unmodified Jurkat cells (Figure 1) that lack the bona fide 237Ab epitope in Tn-PD$\mathrm{PN}$, while the efficacy was completely abolished when the Jurkat cells were restored with wild-type COSMC, indicating the cancer specificity of 237CART cells.

Similarly unexpected was that while 237Ab bound exclusively to Ag104A, the only cancer among the panel that expressed murine PDPN (Figure 2), 237CART cells lysed several different human and mouse cancers not predicted by 237 Ab binding as long as the cell lines were COSMC mutants (Figure 2). By contrast, 237CART cells did not recognize Ag104A when the wild-type COSMC is restored regardless of murine PDPN expression, indicating the exclusive specificity of 237CART cells for the cancer-specific COSMC mutations.

The above findings raised the question of how specific 237Ab was for Tn-PDPN compared to other Tn glycopeptides. Figure 3 (left panel) shows that the 237Ab selectively bound only to cell lines expressing Tn-PDPN. Either knocking out murine PDPN expression or restoration of wild-type COSMC function abrogated 237Ab staining. As controls, an Ab specific for only Tn stained COSMC-mutant cancers regardless of murine PDPN expression, while an Ab specific only for the PDPN peptide backbone epitope stained murine PDPN-positive cancers independent of the COSMC status.

Typically, Ab staining of cell surface antigens is performed at concentrations of approximately $10 \mu \mathrm{g} /$ $\mathrm{mL}(\sim 66.67 \mathrm{nM})$, while higher concentrations of the $237 \mathrm{Ab}$ might have predicted the cross-reactivity of the 237CART cells with human tumors. We therefore examined $237 \mathrm{Ab}$ staining starting at $3000 \mathrm{nM}$. Figure 3 (right 


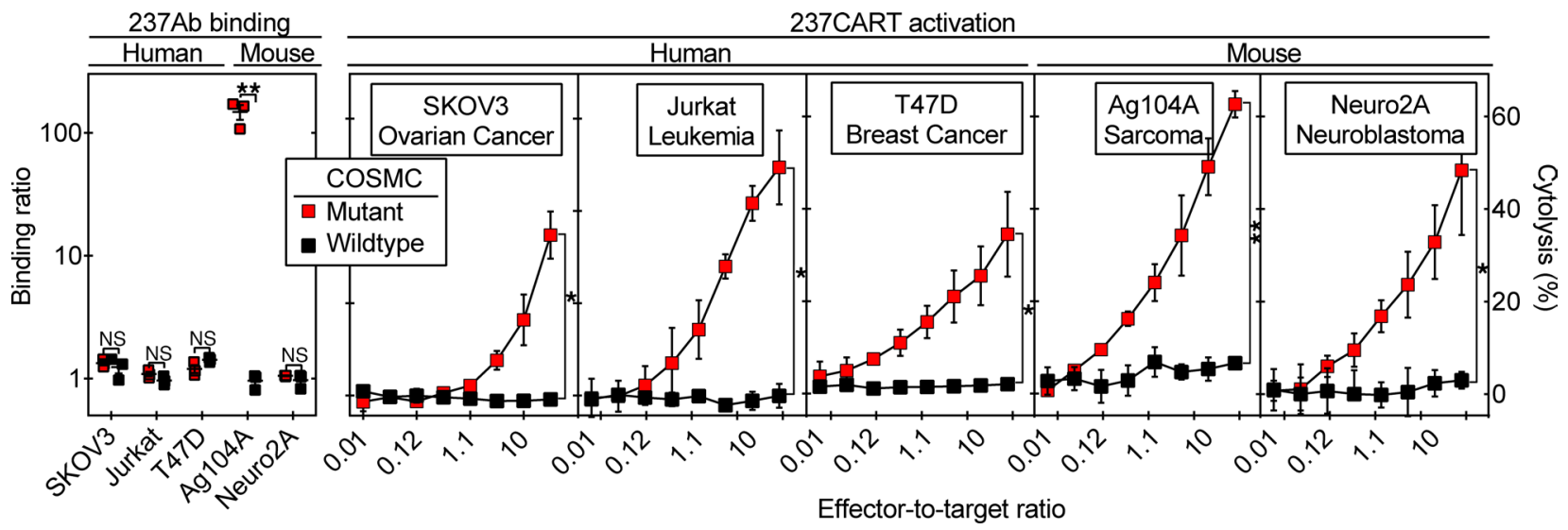

Figure 2. 237CART cells lyse several COSMC-mutant human and murine cancer cell lines not predicted by 237Ab binding. Left panel: 237Ab staining of human and murine cell lines that were mutant or wild-type for COSMC is presented as the binding ratio, as explained in the Methods. Mean \pm SEM, $n=3$ from 3 independent experiments. The significance of the difference in 237Ab staining between the COSMC-mutant and -wild-type variants of each cell line was examined by 2-tailed Student's $t$ test. Right panels: 237CART cells lysed all 3 human cancer cell lines and 2 murine cell lines dependent on COSMC mutation in a 4-hour ${ }^{51} \mathrm{Cr}$ release assay. Mean $\pm \mathrm{SEM}, n=3$ from 3 independent experiments. The significance of the difference between the COSMC-mutant and COSMCwild-type cells at the highest effector-to-target ratio was examined by 2 -tailed Student's $t$ test. ns indicates $P>0.05 ;{ }^{*} P \leq 0.05,{ }^{* *} P \leq 0.01$.

panel) shows that even at this concentration, the staining was negligible. Thus, the 237Ab binding specificity would not have predicted the expanded 237CART cell reactivity with other Tn-glycosylated cancers.

Unlike the 237Ab, 237CART cells did not have exclusive specificity for cells expressing Tn-PDPN Although 237CART cells did recognize COSMC-mutant cancers without murine PDPN, it still reacted with Tn-PDPN, as the 237CART activation level was always lower in the absence of murine PDPN (Figure 4).

To determine whether 237CART cells could indeed react with other peptide sequences while retaining Tn-glycopeptide specificity, we began with systematic single-alanine replacements of the Tn-PDPN motif previously defined by our molecular and crystallographic studies $(6,10)$. Figure 5 (top panels) shows that the $\mathrm{Tn}$ on $\mathrm{Thr}^{77}$ of murine PDPN was essential for 237Ab binding and for 237CART cell activation. Binding of $237 \mathrm{Ab}$ to peptide-coated plates was significantly reduced when the $\mathrm{N}$-terminal glycine was replaced with an alanine. Single-alanine replacement of 2 other amino acids caused a less pronounced reduction. However, none of the single-alanine replacements significantly reduced the activation of 237CART cells alone. We therefore proceeded to multiple-alanine replacements (Figure 5, middle panels). Surprisingly, even replacement of all 5 of the amino acids in the C-terminus of the 237Ab epitope had no significant influence on 237CART cell activation. If, however, the N-terminal glycine was also replaced, 237CART cell activation was then compromised considerably. In contrast, the 237Ab binding was already significantly diminished

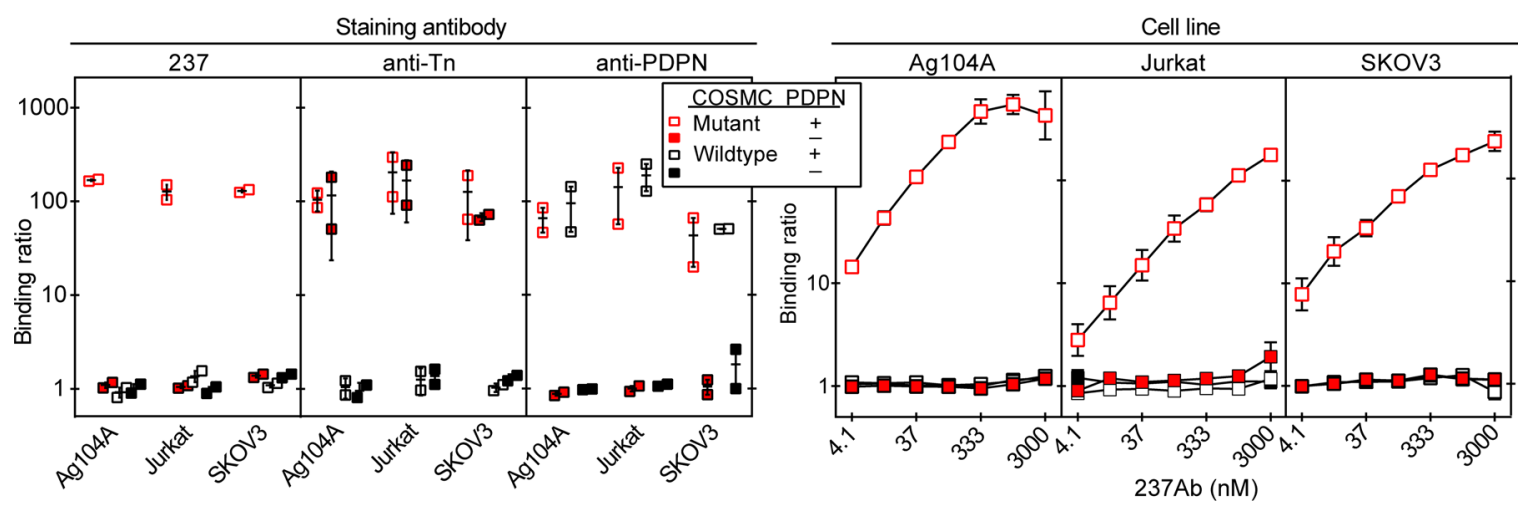

Figure 3. 237Ab only binds to COSMC-mutant cell lines expressing murine PDPN. The COSMC-mutant or -wild-type, PDPN-positive or -negative variants of each parental cell line were generated as described in the Methods. Left panels: 237Ab only stained cell lines that were COSMC-mutant and expressing murine PDPN. The level of binding is represented by the binding ratio (see Methods). Right panels: The exclusive specificity of $237 \mathrm{Ab}$ for murine PDPN-expressing COSMC-mutant cancer cell lines was demonstrated by staining of the cells with serial 3-fold dilutions of 237Ab starting at 3000 $\mathrm{nM}(\sim 450 \mu \mathrm{g} / \mathrm{mL})$. Mean $\pm \mathrm{SEM}, n=2$ from 2 independent experiments. 

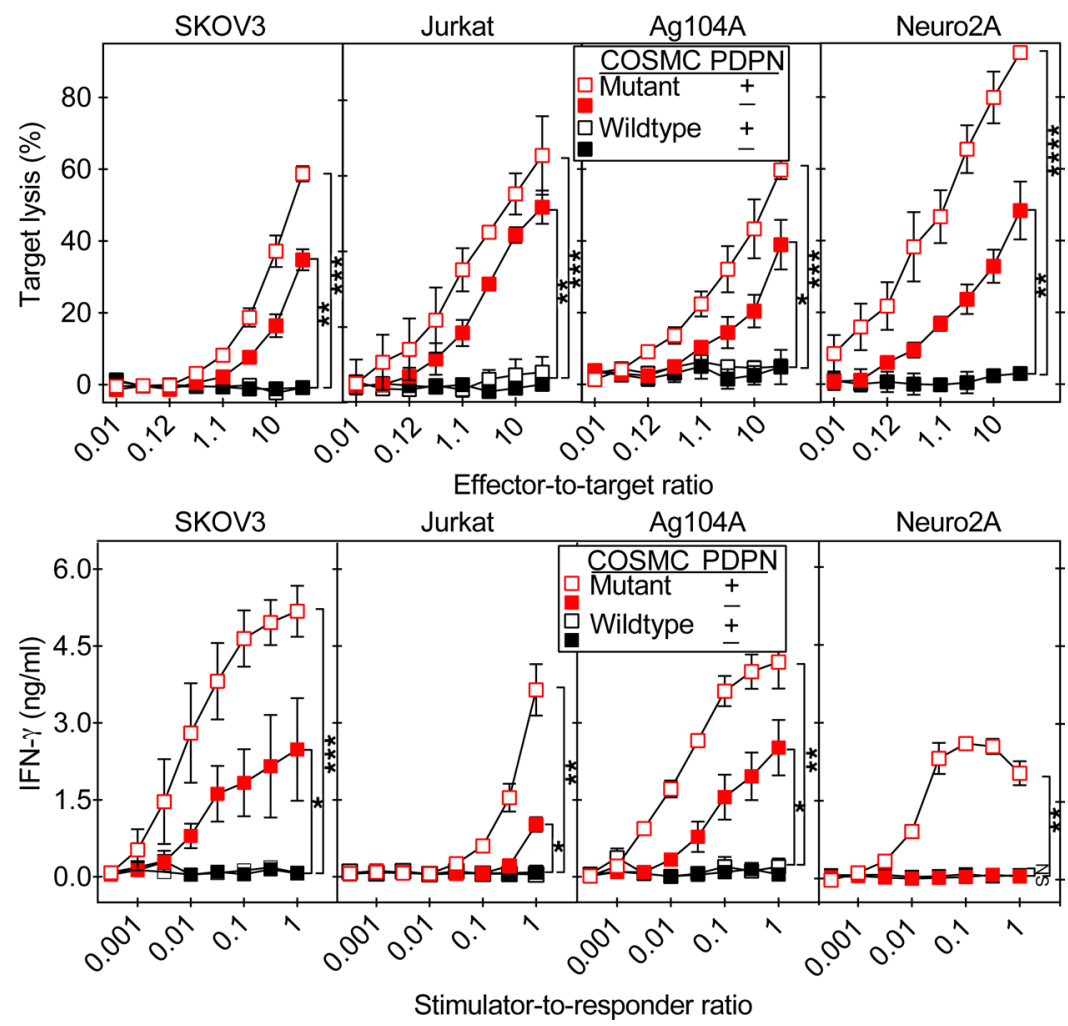

Figure 4. The 237CART recognition of COSMC-mutant cancers prefers, but does not require, Tn-PDPN expression. The COSMC-mutant or -wild-type variants with or without murine PDPN expression of each parental cell line were generated as described in the Methods. The activation of 237CART cells upon recognition of different cell lines was determined by target lysis in a 4 -hour ${ }^{51} \mathrm{Cr}$ release assay (upper panels), and by IFN- $\gamma$ release into the medium after a 24-hour coincubation of 237CART with the target cells (lower panels). Mean \pm SEM, $n=3$ from 3 independent experiments. Significance of the difference between 2 groups as indicated was determined by 2 -tailed Student's $t$ test. ns indicates $P>0.05 ;{ }^{*} P \leq 0.05,{ }^{* *} P \leq 0.01,{ }^{* *} P \leq 0.001$.

after substitution of the N-terminal glycine alone (Figure 5, top left panel). Because the simultaneous alanine replacement of all C-terminal amino acids was permitted, we further investigated what was the minimal length of the C-terminal peptide required for 237CART cell activation. Figure 5 (bottom panels) shows that the shortening of the peptide always had a more significant influence on 237Ab binding than on 237CART cell activation, while the reduction of more than 4 amino acids virtually abrogated all reactivity. The above experiments demonstrate a greater permissiveness for 237CART cell activation than 237Ab binding with alanine replacements and shortening of the 237Ab binding motif within the murine PDPN.

Because disseminated, established Jurkat leukemia cells were effectively eliminated by 237CART cells even in the absence of murine PDPN, we tested several other Tn-glycopeptide antigens found to be expressed on Jurkat cells $(11,12)$. Figure 6A shows that 237Ab binds only to Tn-PDPN, whereas 237CART cells react at different levels with several other Tn glycopeptides expressed by Jurkat cancer cells. This result revealed that 237CART cells can recognize multiple different Tn glycopeptides on a single Jurkat cell, which may prevent escape by ALVs and thereby contribute to long-term disease-free survival.

As a test for the generality of our observation that a CAR derived from a Tn-glycopeptide-specific $\mathrm{Ab}$ would react with more Tn glycopeptides than predicted by the corresponding Ab, we examined 5E5CART cells derived from $5 \mathrm{E} 5 \mathrm{Ab}$, which was generated by immunizing mice with Tn-glycosylated human mucin 1 (Tn-MUC1) $(13,14)$. While 5E5Ab only recognized Tn-MUC1 (Figure 6B), 5E5CART cells also recognized Tn-PDPN (Figure 6B), which was not predicted by 5E5Ab binding. The pattern of cross-reactivity was different between 237CART and 5E5CART cells, since 5E5CART cells were not activated by several Tn glycopeptides that activated 237CART (Figure 6A). Consistent with the notion that 5E5CART cells recognized more Tn glycopeptides than that predicted by $5 \mathrm{E} 5 \mathrm{Ab}$ binding, we also found that 5E5CART not only killed cancers expressing Tn-MUC1 as reported (15), but also COSMC-mutant cancers that expressed neither MUC1 nor murine PDPN (Figure 7), supporting the generality of our discovery made with 237CART cells. 


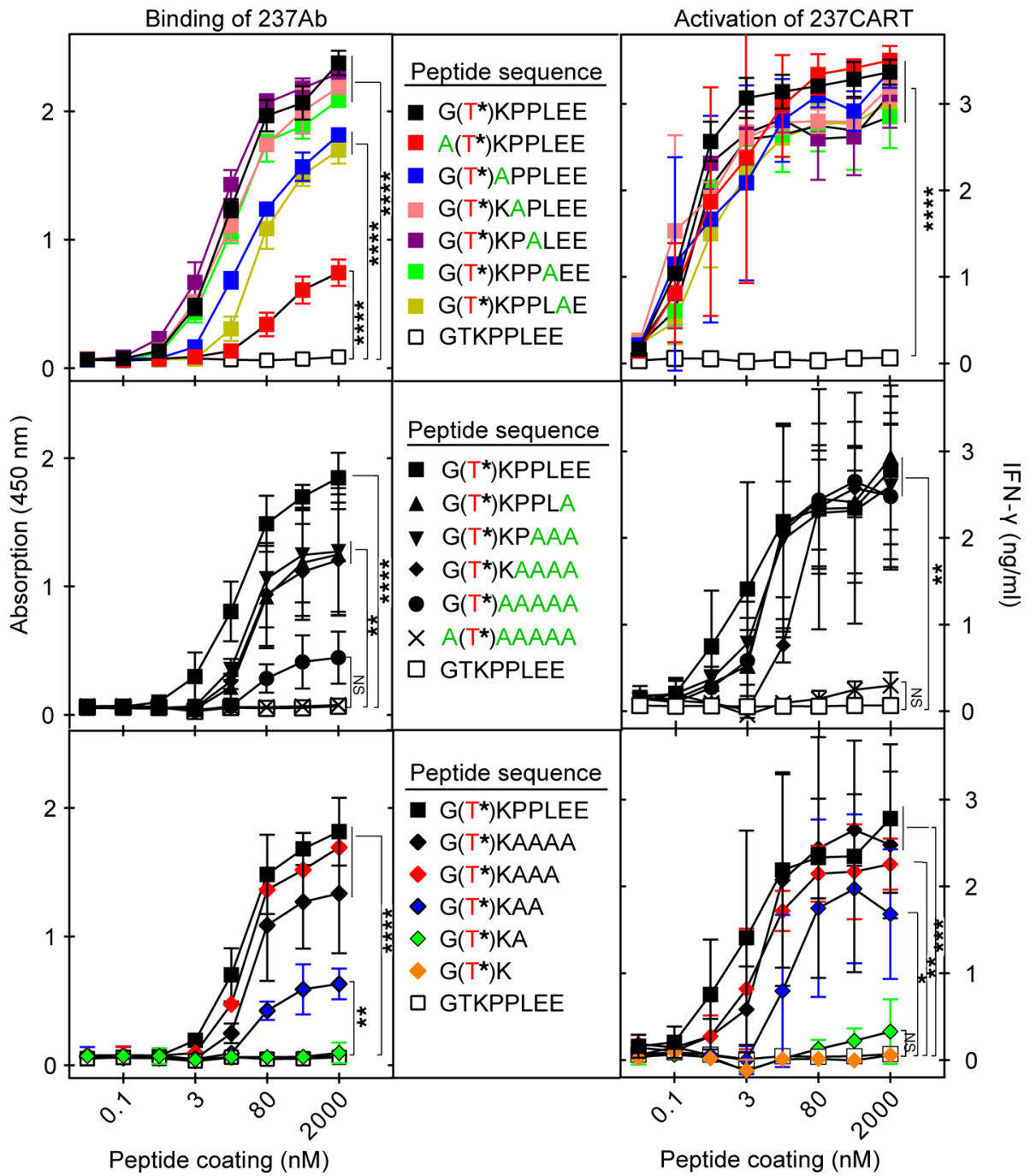

Figure 5. Recognition by 237CART cells is more permissive to amino acid residue substitutions and truncations of the Tn-glycopeptide epitope than that by 237Ab. Biotinylated peptides immobilized on a streptavidin-coated plate surface at the indicated coating concentrations were tested for binding by 237Ab measured by light absorption at $450 \mathrm{~nm}$, or stimulation of 237CART cells measured by the level of IFN- $\gamma$ secretion after 24 hours of coincubation. The Tn-glycosylated Thr ${ }^{77}$ of murine PDPN is labeled in red, while the alanine scanning of the original murine PDPN sequence is labeled in green. Top panel: Alanine scanning of the 237Ab-binding epitope, 1 amino acid residue at a time. Middle panel: Alanine scanning of the 237Ab-binding epitope in murine PDPN with an increasing number of residues at a time. Bottom panel: The gradual truncation of the 237Ab-binding epitope from the C-terminus. Mean \pm SEM, $n=3$ from 3 independent experiments. The significance of the difference between each group at the highest peptide coating concentration in comparison to that of the non-Tn-glycosylated GTKPPLEE group was examined by 1-way ANOVA followed by Dunnett's test. ns indicates $P>0.05 ;{ }^{*} P \leq 0.05,{ }^{* *} P \leq 0.01,{ }^{* * *} P \leq 0.001,{ }^{* * *} P \leq 0.0001$.

\section{Discussion}

Our study shows that multiple different cancer-specific antigens on a single cancer cell can be targeted by 237CART cells. This broad reactivity to Tn-glycosylated antigens was not predicted by the specificity of the $237 \mathrm{Ab}$. Two complementary explanations present themselves. One is that the 237Ab single-chain variable fragment $(\mathrm{scFv})$ used in the 237CAR construction has a different specificity than the original $237 \mathrm{Ab}$ due to conformational changes. Such a model of broadened specificity is consistent with previous reports that some proteins such as enzymes and Abs may have polyfunctional combining regions for recognition of structurally related ligands $(16,17)$.

A second explanation is that the avidity of CAR binding was increased because multiple CARs concentrated at the immunological synapse during CART cell activation. This may have enhanced the sensitivity of the CART cells to recognize some weak-interacting, suboptimal Tn-glycopeptide epitopes (18). Flow cytometry nor- 

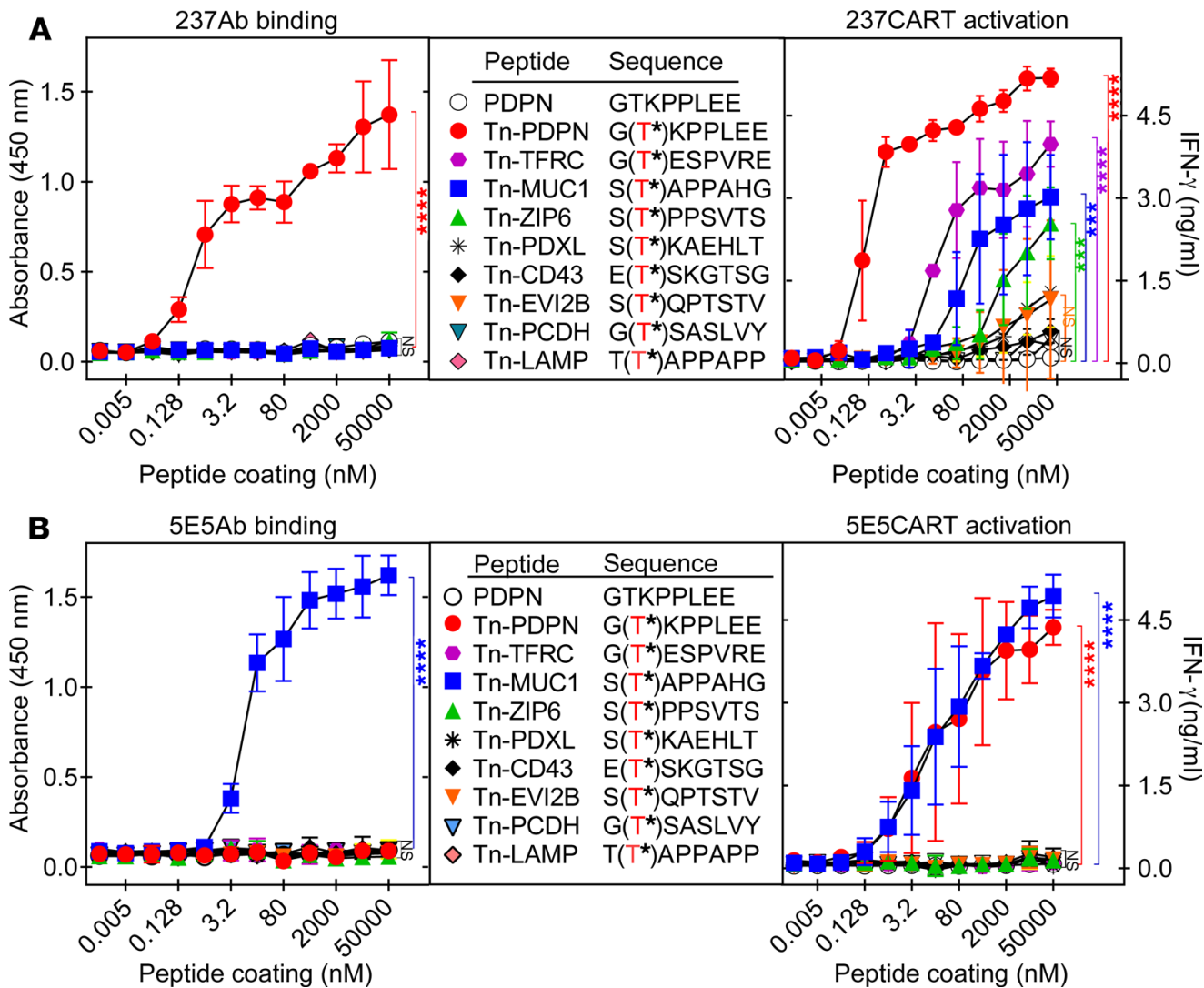

Figure 6. CART cells derived from a Tn-glycopeptide-specific Ab can recognize multiple different Tn-glycopeptide antigens. Biotinylated Tn glycopeptides containing epitopes found in Jurkat cancer cells were immobilized on a streptavidin-coated plate surface, and tested for recognition by 237Ab or 5E5Ab binding, or activation of 237CART or 5E5CART cells, as described in Figure 5. (A) 237CART cells made from the murine Tn-PDPN-specific $237 \mathrm{Ab}$ recognized multiple different $\mathrm{Tn}$-glycopeptide antigens from Jurkat cancer cells not predicted by the $237 \mathrm{Ab}$ binding. (B) 5E5CART cells made from the Tn-MUC1-specific 5E5Ab exhibited similar reactivity upon recognition of Tn-MUC1 or Tn-PDPN, while 5E5Ab only had detectable binding to Tn-MUC1. Mean \pm SEM, $n=3$ from 3 independent experiments. The significance of the difference between each group at the highest peptide coating concentration in comparison to that of the non-Tn-glycosylated GTKPPLEE group was examined by 1-way ANOVA followed by Dunnett's test. ns indicates $P>0.05 ;{ }^{*} P \leq 0.05,{ }^{* *} P \leq 0.01,{ }^{* *} P \leq 0.001,{ }^{* * *} P \leq 0.0001$.

mally requires binding of more than 1,000 Ab molecules per cell to generate a meaningful signal. By contrast, target lysis by $\mathrm{T}$ cells can be mediated by as few as 1-10 TCR engagements, or 100-200 CAR engagements per cell (19-22). Consistent with this notion is that 237CART cells are more sensitive in detecting Tn-PDPN than bispecific antibodies made with $237 \mathrm{scFv}$ and an anti-CD3 scFv (18). The sensitivity of CART cells to detect low levels of antigens may lead to serious toxicities when such CART cells recognize self-antigens expressed on normal tissue $(23,24)$. Fortunately, in the case of the CART cells derived from the Tn-glycopeptide-specific $237 \mathrm{Ab}$, cross-reactivity has only been detected with other Tn-glycopeptide antigens. These antigens depend on a loss-of-function mutation in COSMC that makes recognition highly cancer specific. The molecular basis of this observation may be explained by our previous crystallographic analyses of 237Ab showing that $237 \mathrm{Ab}$ uses a pocket coded entirely by germline regions of the $\mathrm{Ab}$ to envelop the $\mathrm{Tn}$ inside the pocket (10), making the binding entirely dependent on the presence of the carbohydrate moiety of the epitope. In normally glycosylated cells, $\mathrm{Tn}$ is hidden by the extended O-linked glycosylation and therefore no longer fits inside the pocket. There are also some additional interactions between the $237 \mathrm{CDRs}$ and the peptide backbone that envelop $\mathrm{Tn}$ inside the pocket, which may explain the preference of 237CART cells for some Tn-glycopeptide antigens. Attempts to crystallize $5 \mathrm{E} 5 \mathrm{Ab}$ have not yet been successful. We do not know whether $5 \mathrm{E} 5 \mathrm{Ab}$ would also envelop Tn inside a pocket, or whether the interaction is more superficial, as is the case for the AR20.5 Ab (25).

Aberrant Tn expression can be found in various types of human cancers. Immunohistochemically, over $80 \%$ of human breast cancers (13) and over $85 \%$ of human ovarian cancers are positive for $5 \mathrm{E} 5 \mathrm{Ab}$ 

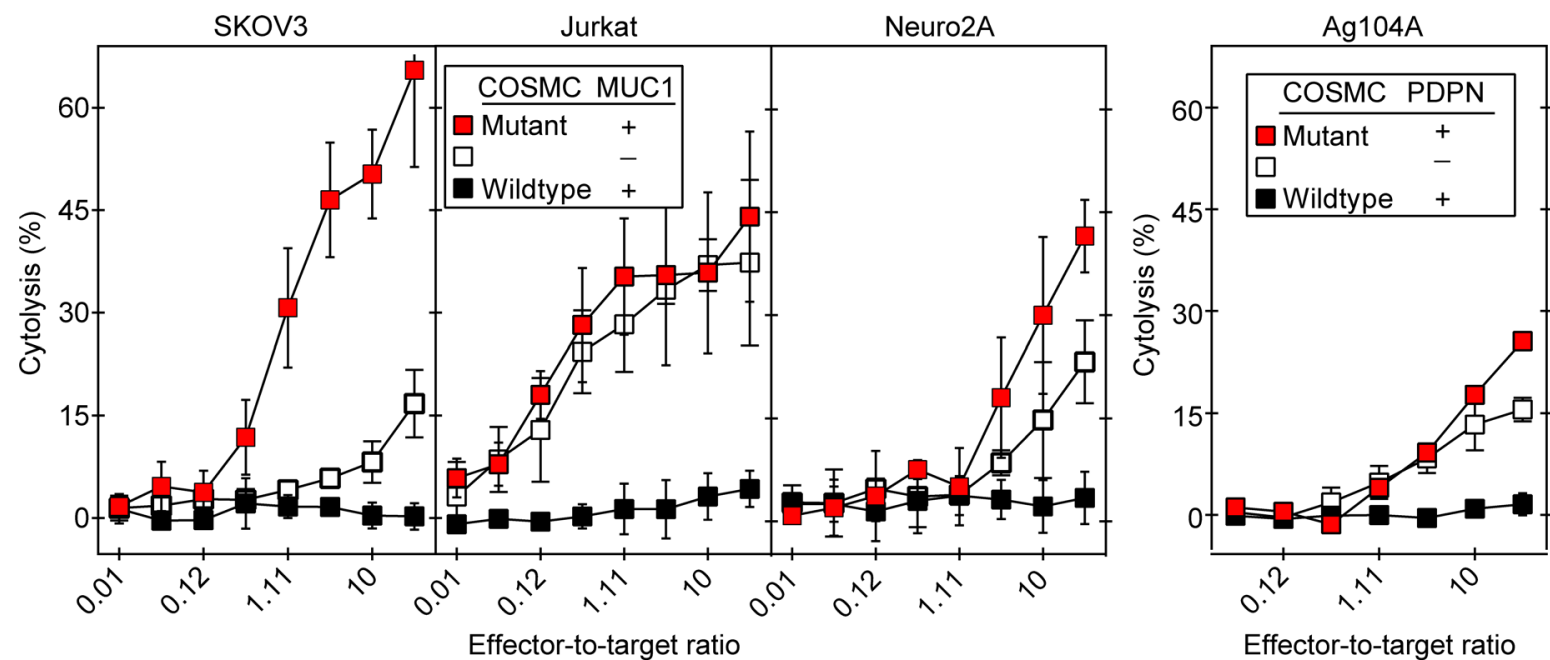

Figure 7. 5E5CART cells preferentially, but not exclusively, recognize cancer cells expressing Tn-MUC1 or murine Tn-PDPN. The COSMC-mutant or -wildtype, MUC1/PDPN-positive or -negative variants of each parental cell line were generated as described in the Methods. 5E5CART cells were tested for Iytic activity in a 4-hour ${ }^{51} \mathrm{Cr}$ release assay upon recognition of human and murine cancer cell lines in the presence or absence of Tn-MUC1 expression (left panels) or murine Tn-PDPN expression (right panel). Mean \pm SEM, $n=2$ from 2 independent experiments.

staining (26). COSMC mutations (27) can be found in $1 \%-6 \%$ of human cancers (Figure 8 ), and are one major mechanism that leads to Tn expression on the surface of cancer cells $(6,28,29)$. Altered expression or localization of different types of GalNAc transferases can lead to Tn glycosylation as well (30-34). Epigenetic silencing of COSMC and/or T-synthase expression is another mechanism that results in stable Tn expression $(35,36)$. Surface Tn expression has been exclusively found in cancers, except for a rare form of acquired hemolytic anemia (27). Thus, the exclusive cancer specificity and the broad recognition of various Tn-glycopeptide antigens make 237CART cells a desirable candidate for treating human cancers with Tn expression on the cell surface.

\section{Methods}

Mice. C57BL/6-Rag1-/- (B6.129S7-Rag1tmiMom/J), OT-1 (C57BL/6-Tg(TcraTcrb)1100Mjb/J), and NSG

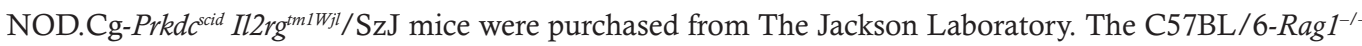
mice were subsequently bred with the OT-1 mice to generate the OT-1-Rag1/- mice (37).

Cell lines. Ag104A and Neuro2A are spontaneous murine cancer cell lines lacking COSMC function due to somatic mutations in the Cosmc gene (6). The COSMC wild-type variants were generated by retroviral transduction of wild-type Cosmc. Ag104A naturally expresses a high level of murine PDPN. The murine PDPN-negative Ag104A variant was made by CRISPR-Cas9, knocking out Pdpn using the guiding sequence GATATTGTGACCCCAGGTAC. Neuro2A naturally does not express murine PDPN nor MUC1. The murine PDPN-positive Neuro2A variant was prepared by retroviral transduction and the MUC1-positive variant was prepared by plasmid transfection. Jurkat E6-1 is a human T cell leukemia carrying a null mutation of $\operatorname{COSMC}(28)$ and does not express murine PDPN. The COSMC wild-type Jurkat E6-1 variant was generated by lentiviral transduction and the murine PDPN-expressing variant was generated by retroviral transduction. The MUC1-negative variant was generated by CRISPR-Cas9 knockout, using the guiding sequence TGAAGCTGGTTCCGTGGCCG. SKOV3 and T47D are human cancer cell lines with COSMC function. COSMC was knocked out by CRISPR-Cas9 using the guiding sequence TCACTATGCTAGGACAC. $M U C 1$ was knocked out by CRISPR-Cas9 using the guiding sequence TGAAGC TGGTTCCGTGGCCG. The murine PDPN-expressing variant of SKOV3 was generated by retroviral transduction. All cancer cell lines except Jurkat E6-1 and its variants were maintained in DMEM. Jurkat E6-1 and its variants were maintained in RPMI1640. All culture medium was supplemented with $10 \%$ FCS (Gemini).

Antibodies. The $237 \mathrm{mAb}$ was generated in-house from a hybridoma cell line, as described previously $(5,6)$. The Tn-specific mAb 5F4 was prepared from a hybridoma cell line, as described previously (38), as was the MUC1-specific mAb 5E10, as described previously (14). The murine PDPN-specific mAb PA2.26 


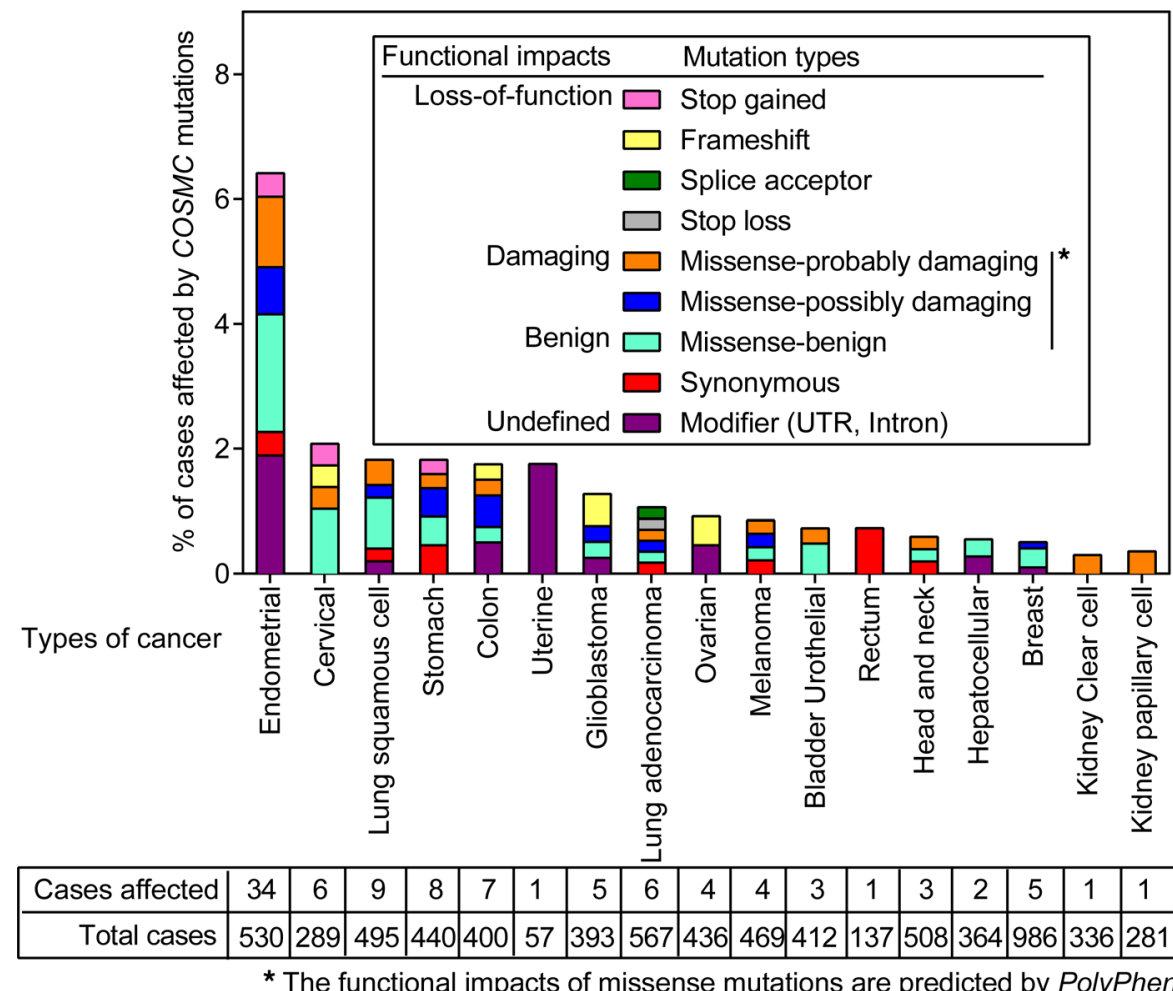

Figure 8. The frequency and the type of COSMC mutations in human cancers across different cancer types. COSMC mutations across different cancer types were extracted from The Cancer Genome Atlas (TCGA) database (https://portal. gdc.cancer.gov). The types of mutations are indicated in the legend within the figure. The number of cases affected by COSMC mutations of the total number of cases analyzed in different cancer types is indicated in the box below the figure.

was a gift from Miguel Quintanilla (Universidad Autónoma de Madrid, Madrid, Spain) (39). The APC $\mathrm{F}\left(\mathrm{ab}^{\prime}\right) 2$ anti-mouse $\operatorname{IgG}(\mathrm{H}+\mathrm{L})$ used for secondary $\mathrm{Ab}$ staining for detection of primary $\mathrm{Ab}$ binding is a polyclonal Ab from Southern Biotech (catalog 1080-11L). The Alexa Fluor 647-labeled polyclonal F(ab')2 anti-mouse $\mathrm{IgG}(\mathrm{H}+\mathrm{L})$ (Invitrogen, catalog A21237) was used for detection of CAR expression on the T cell surface. For stimulation of mouse splenocytes for retroviral transduction, $1.5 \mu \mathrm{g} / \mathrm{mL}$ anti-CD3 $\mathrm{Ab}$ (clone 145-2C11, University of Chicago, Frank Fitch Monoclonal Ab Facility) and $0.5 \mu \mathrm{g} / \mathrm{mL}$ anti-CD28 $\mathrm{Ab}$ (clone 37.51, BioLegend, catalog 102116) were coated on culture dishes overnight at $4^{\circ} \mathrm{C}$

CRISPR-Cas9 knockout. For CRISPR-Cas9-mediated gene knockouts, the guiding sequences were designed using the sgRNA designer web tool from the Broad Institute (https://portals.broadinstitute.org/gpp/public/ analysis-tools/sgrna-design), ordered from Integrated DNA Technologies, Inc, and subcloned into vector pSPCas9(BB)-2A-GFP (PX458, Addgene) as described previously (40). Cell lines were transfected with the plasmid by calcium phosphate precipitation, and the plasmid-transfected cells were sorted based on GFP expression. From the CRISPR-Cas9 vector-transfected cells, the COSMC-knockout cells were further sorted based on Tn expression by $5 \mathrm{~F} 4 \mathrm{mAb}$ staining, and MUC1-knockout cells were sorted based on MUC1 staining by $5 \mathrm{E} 10 \mathrm{mAb}$.

Flow cytometry. Samples were incubated with primary Abs followed by secondary APC-goat antimouse IgG(H+L) polyclonal Ab (Southern Biotech). Cytometry data were collected on an LSR II (BD Bioscience) and analyzed by Flowjo (Tree Star). The binding ratio represents the value of median fluorescence intensity (MFI) of a cell line stained with primary and secondary Abs divided by the MFI of the staining with the secondary $\mathrm{Ab}$ only.

T cell transduction. 237CAR, 5E5CAR, or CD19CAR cells were retrovirally transduced into splenocytes isolated from OT-1-Rag ${ }^{-1-}$ mice, as described previously (37).

Immobilization of peptides on streptavidin-coated plates. To coat immunosorbent 96-well plates (Sigma-Aldrich, catalog M9410) with streptavidin, $50 \mu \mathrm{L}$ of $10 \mu \mathrm{g} / \mathrm{mL}$ streptavidin (Sigma-Aldrich, catalog 189730) in double-distilled water was added to each well and left to dry on the plate at room temperature overnight. The unbound surface was blocked by ELISA diluent (eBioscience, catalog 00-4202-56) for 1 hour at room 
temperature. After washing 3 times with PBST (PBS containing 0.5\% Tween 20 [Sigma-Aldrich, catalog $\mathrm{P} 1379]), 50 \mu \mathrm{L}$ of biotinylated peptides in ELISA diluent at concentrations as indicated in the figures were added to each well of the streptavidin-coated plate, incubated for 2 hours at room temperature, and then washed again twice with PBST before being used for CART cell activation or Ab binding studies.

Cytokine release assay. CART cells $\left(1 \times 10^{4}\right)$ were cocultured with target cells at the indicated stimulator-to-responder ratio, or with immobilized peptides coated on the plate surface for 24 hours. The level of IFN- $\gamma$ release into the medium was detected by ELISA (Ready-SET-Go!, eBioscience, catalog 50-173-21). The level of light absorbance at $450 \mathrm{~nm}$ was read by a VERSAmax microplate reader (Molecular Devices).

Cytotoxicity assay. The capability of CART cells to lyse target cells was evaluated in a 4-hour ${ }^{51} \mathrm{Cr}$ release assay, as described previously (37). Target cells were incubated with $25 \mu$ Ci sodium chromate-51 (Perkin Elmer) in the culture medium for 1 hour, washed 3 times with culture medium, and resuspended at $1 \times$ $10^{5} \mathrm{cell} / \mathrm{mL}$. Fifty microliters of the target cell suspension $(n=5,000)$ was mixed with $50 \mu \mathrm{L}$ of effector CART cell suspension in each well of a U-bottomed 96-well cell-culture plate (Thermo Fisher Scientific) at the indicated effector-to-target ratios. ZAP-OGLOBIN II lytic reagent was used as positive control following the manufacturer's instruction (Beckman Coulter, 7546138). No effector cells nor any lytic reagent was added to the target cells for spontaneous release control. After 4 hours of coincubation, $100 \mu \mathrm{L}$ of supernatant from each well was collected and transferred into glass tubes (VWR, $6 \times 50 \mathrm{~mm}$ ). Target killing was evaluated by the level of radioactivity released into the medium measured by an automatic $\gamma$-counter (Titertek). The percentage of target lysis was calculated by the following formula: (experimental values spontaneous release values)/(maximum release values - spontaneous release values $) \times 100$.

Ab binding to peptides. Fifty microliters of $10 \mu \mathrm{g} / \mathrm{mL} 237 \mathrm{Ab}$ or $5 \mathrm{E} 5 \mathrm{Ab}$ was added to each well containing immobilized peptides and incubated for 1 hour at room temperature. Ab binding was detected by sandwich ELISA according to the manufacturer's protocol (eBioscience, catalog 50-173-21) and absorbance at $450 \mathrm{~nm}$ was detected with a VERSAmax microplate reader.

Bioluminescence imaging. Jurkat E6-1 cells and its variants were modified to express green click beetle luciferase, $5 \times 10^{6}$ cells were injected through tail vein, and disease progression was monitored by weekly bioluminescence imaging on a Xenogen IVIS-200 Spectrum camera (Perkin Elmer). D-luciferin, potassium salt (Goldbio, catalog LUCK-100) was intraperitoneally injected according to the manufacturer's instruction 15 minutes before imaging.

Statistics. Data were analyzed using Prism software (GraphPad). Error bars in the figures indicate SEM of at least 2 independent experiments. For the comparison of 2 groups where the data were normally distributed, a 2-tailed Student's $t$ test was used. The significance level of the difference between the survival of animals from the different treatment groups was determined by the log-rank Mantel-Cox test. The $P$ values are indicated in the figure legends (ns indicates not significant, $P>0.05 ;{ }^{*} P \leq 0.05$, ${ }^{* *} P \leq 0.01$, ${ }^{* *} P \leq 0.001,{ }^{* * * *} P \leq 0.0001$ ).

Study approval. All animal experiments followed federal guidelines, approved by the Institutional Animal Care and Use Committee (IACUC) and the Institutional Biosafety Committee (IBC) at the University of Chicago.

\section{Author contributions}

YH, KS, HMS, and H. Schreiber designed the project. YH, KS, SPW, and FW conducted experiments. AP, PS, JZ, CS, UM, and MS provided essential help for the experiments. ML, SCM, DMK, MS, HC, CHJ, AP, FW, H. Steiner, and KS contributed to writing the manuscript.

\section{Acknowledgments}

This study was supported by NIH grants R01-CA22677, R01-CA37156, and R01-CA226983; the Gerald O. Mann Foundation/Harriet and Allan Wulfstat, Trustees; a gift from Janet D. Rowley to KS and HS; the Cancer Research Foundation; the Lundbeck Foundation (R221-2016-438); Læge Sofus Carl Emil Friis og hustru Olga Doris Friis' Legat; and the Danish National Research Foundation (DFF-4004-00397B). The frequency and the types of COSMC mutations among different cancer types in Figure 8 were generated based on data acquired from the Cancer Genome Atlas (TCGA) research network: https://www.cancer. gov/tcga. We thank Boris Engels and Cecelia Lai for helping with CAR construction and transduction, and Patrick Moore for helping with quantifying glycopeptides, and Eric Seidel for his advice on the CRISPR-Cas9 system. We also thank Sandra J. Gendler for her advice on MUC1. 
Address correspondence to: Hans Schreiber, The University of Chicago; Department of Pathology; 5841 S. Maryland Avenue, Chicago, Illinois 60637, USA. Phone: 773.702.9204, Email: hszz@uchicago.edu.

1. June CH, Sadelain M. Chimeric Antigen Receptor Therapy. N Engl J Med. 2018;379(1):64-73.

2. Hegde $\mathrm{M}$, et al. Tandem CAR T cells targeting HER2 and IL13R $\alpha 2$ mitigate tumor antigen escape. J Clin Invest. 2016;126(8):3036-3052.

3. Roybal KT, et al. Precision Tumor Recognition by T Cells With Combinatorial Antigen-Sensing Circuits. Cell. 2016;164(4):770-779.

4. Brentjens RJ, et al. Eradication of systemic B-cell tumors by genetically targeted human T lymphocytes co-stimulated by CD80 and interleukin-15. Nat Med. 2003;9(3):279-286.

5. Ward PL, Koeppen H, Hurteau T, Schreiber H. Tumor antigens defined by cloned immunological probes are highly polymorphic and are not detected on autologous normal cells. J Exp Med. 1989;170(1):217-232.

6. Schietinger A, et al. A mutant chaperone converts a wild-type protein into a tumor-specific antigen. Science. 2006;314(5797):304-308.

7. Brentjens RJ, et al. Genetically targeted T cells eradicate systemic acute lymphoblastic leukemia xenografts. Clin Cancer Res. 2007;13(18 Pt 1):5426-5435.

8. Davila ML, Kloss CC, Gunset G, Sadelain M. CD19 CAR-targeted T cells induce long-term remission and B Cell Aplasia in an immunocompetent mouse model of B cell acute lymphoblastic leukemia. PLoS One. 2013;8(4):e61338.

9. Steentoft C, et al. Characterization of an immunodominant cancer-specific O-glycopeptide epitope in murine podoplanin (OTS8). Glycoconj J. 2010;27(6):571-582.

10. Brooks CL, et al. Antibody recognition of a unique tumor-specific glycopeptide antigen. Proc Natl Acad Sci U S A. 2010;107(22):10056-10061.

11. Mukherjee P, Tinder TL, Basu GD, Gendler SJ. MUC1 (CD227) interacts with lck tyrosine kinase in Jurkat lymphoma cells and normal T cells. J Leukoc Biol. 2005;77(1):90-99.

12. Steentoft C, et al. Mining the O-glycoproteome using zinc-finger nuclease-glycoengineered SimpleCell lines. Nat Methods. 2011;8(11):977-982.

13. Sørensen AL, et al. Chemoenzymatically synthesized multimeric Tn/STn MUC1 glycopeptides elicit cancer-specific antiMUC1 antibody responses and override tolerance. Glycobiology. 2006;16(2):96-107.

14. Tarp MA, et al. Identification of a novel cancer-specific immunodominant glycopeptide epitope in the MUC1 tandem repeat. Glycobiology. 2007;17(2):197-209.

15. Posey AD, et al. Engineered CAR T Cells Targeting the Cancer-Associated Tn-Glycoform of the Membrane Mucin MUC1 Control Adenocarcinoma. Immunity. 2016;44(6):1444-1454.

16. Glazer AN. On the prevalence of "nonspecific" binding at the specific binding sites of globular proteins. Proc Natl Acad Sci U S A. 1970;65(4):1057-1063.

17. Richards FF, Konigsberg WH, Rosenstein RW, Varga JM. On the specificity of antibodies. Science. 1975;187(4172):130-137.

18. Stone JD, Aggen DH, Schietinger A, Schreiber H, Kranz DM. A sensitivity scale for targeting T cells with chimeric antigen receptors (CARs) and bispecific T-cell Engagers (BiTEs). Oncoimmunology. 2012;1(6):863-873.

19. Purbhoo MA, Irvine DJ, Huppa JB, Davis MM. T cell killing does not require the formation of a stable mature immunological synapse. Nat Immunol. 2004;5(5):524-530.

20. Huse M, et al. Spatial and temporal dynamics of T cell receptor signaling with a photoactivatable agonist. Immunity. 2007;27(1):76-88

21. Huang $\mathrm{J}$, et al. A single peptide-major histocompatibility complex ligand triggers digital cytokine secretion in CD4(+) $\mathrm{T}$ cells. Immunity. 2013;39(5):846-857.

22. Han A, Glanville J, Hansmann L, Davis MM. Linking T-cell receptor sequence to functional phenotype at the single-cell level. Nat Biotechnol. 2014;32(7):684-692.

23. Morgan RA, Yang JC, Kitano M, Dudley ME, Laurencot CM, Rosenberg SA. Case report of a serious adverse event following the administration of T cells transduced with a chimeric antigen receptor recognizing ERBB2. Mol Ther. 2010;18(4):843-851.

24. Bedoya F, Frigault MJ, Maus MV. The Flipside of the Power of Engineered T Cells: Observed and Potential Toxicities of Genetically Modified T Cells as Therapy. Mol Ther. 2017;25(2):314-320.

25. Movahedin M, Brooks TM, Supekar NT, Gokanapudi N, Boons GJ, Brooks CL. Glycosylation of MUC1 influences the binding of a therapeutic antibody by altering the conformational equilibrium of the antigen. Glycobiology. 2017;27(7):677-687.

26. Van Elssen CH, et al. Expression of aberrantly glycosylated Mucin-1 in ovarian cancer. Histopathology. 2010;57(4):597-606.

27. Ju T, Cummings RD. Protein glycosylation: chaperone mutation in Tn syndrome. Nature. 2005;437(7063):1252.

28. Ju T, Cummings RD. A unique molecular chaperone Cosmc required for activity of the mammalian core 1 beta 3-galactosyltransferase. Proc Natl Acad Sci U S A. 2002;99(26):16613-16618.

29. Ju T, et al. Human tumor antigens Tn and sialyl Tn arise from mutations in Cosmc. Cancer Res. 2008;68(6):1636-1646

30. Gill DJ, Clausen H, Bard F. Location, location, location: new insights into O-GalNAc protein glycosylation. Trends Cell Biol. 2011;21(3):149-158.

31. Guda $\mathrm{K}$, et al. Inactivating germ-line and somatic mutations in polypeptide $\mathrm{N}$-acetylgalactosaminyltransferase 12 in human colon cancers. Proc Natl Acad Sci U S A. 2009;106(31):12921-12925.

32. Guo JM, Chen HL, Wang GM, Zhang YK, Narimatsu H. Expression of UDP-GalNAc:polypeptide N-acetylgalactosaminyltransferase-12 in gastric and colonic cancer cell lines and in human colorectal cancer. Oncology. 2004;67(3-4):271-276.

33. Park JH, Katagiri T, Chung S, Kijima K, Nakamura Y. Polypeptide N-acetylgalactosaminyltransferase 6 disrupts mammary acinar morphogenesis through O-glycosylation of fibronectin. Neoplasia. 2011;13(4):320-326.

34. Taniuchi K, et al. Overexpression of GalNAc-transferase GalNAc-T3 promotes pancreatic cancer cell growth. Oncogene. 2011;30(49):4843-4854.

35. Mi R, et al. Epigenetic silencing of the chaperone Cosmc in human leukocytes expressing tn antigen. J Biol Chem. 
2012;287(49):41523-41533.

36. Radhakrishnan P, et al. Immature truncated O-glycophenotype of cancer directly induces oncogenic features. Proc Natl Acad Sci U S A. 2014;111(39):E4066-E4075.

37. Leisegang M, et al. Eradication of Large Solid Tumors by Gene Therapy with a T-Cell Receptor Targeting a Single Cancer-Specific Point Mutation. Clin Cancer Res. 2016;22(11):2734-2743.

38. Kjeldsen T, Clausen H, Hirohashi S, Ogawa T, Iijima H, Hakomori S. Preparation and characterization of monoclonal antibodies directed to the tumor-associated O-linked sialosyl-2----6 alpha-N-acetylgalactosaminyl (sialosyl-Tn) epitope. Cancer Res. 1988;48(8):2214-2220

39. Gandarillas A, Scholl FG, Benito N, Gamallo C, Quintanilla M. Induction of PA2.26, a cell-surface antigen expressed by active fibroblasts, in mouse epidermal keratinocytes during carcinogenesis. Mol Carcinog. 1997;20(1):10-18.

40. Ran FA, Hsu PD, Wright J, Agarwala V, Scott DA, Zhang F. Genome engineering using the CRISPR-Cas9 system. Nat Protoc. 2013;8(11):2281-2308. 